\title{
An Automated Approach to Assessment Management of Higher Education Institutions
}

\author{
NICHOLAS HARKIOLAKIS, PhD \\ Information Technology \\ Hellenic American University \\ 12 Kaplanon Street, Athens 10680 \\ GREECE
}

\begin{abstract}
This paper presents the development of a knowledge management tool that addresses the needs of the higher education institutions in terms of their self evaluation process. The tool aims at bridging the gap between the collection, handling and distribution of data by engaging the various stakeholders in a dialogue and action to improve outcomes. It was developed and modelled according to the quality assurance standards and processes adopted by the oldest American regional accreditation association the New England Association of Schools \& Colleges Inc. (NEASC). Conceptually the tool is focused on the six interrogatives of the English language: What, Where, When, Who, How and Why. The questions raised are grouped and addressed in three areas, Description, Appraisal and Projection, according to the format suggested by NEASC.
\end{abstract}

Key-Words: - Knowledge management, self evaluation, higher education institutions

\section{Introduction}

Knowledge management can be perceived as a set of practices that helps to improve the use and sharing of data and information in decision-making. These practises are gaining widespread acceptance in the field of education where the institutions as seeking ways to capture and organize their internal organizational knowledge in order to improve services and outcomes [1]. More broadly knowledge management can be thought of as a framework or an approach that enables students, faculty, and staff to develop better ways to transform knowledge into effective decision-making and action [2].

Higher education institution administrators are forced to adapt to external demands for quality assessment. In light of the external and internal demands for accountability and improvement in education, institutions and systems at all levels are seeking to understand how they can more effectively collect, disseminate, and share information. As part of regional and specialized accreditation association requirements institutions are expected to provide evidence of clear and appropriate educational objectives, planning and implementation at institutional and program levels. Associations expect institutions to document a process of review that includes the collection and use of data for ongoing self analysis. Alignment between the institution's mission, purposes, and objectives and the actual outcomes of programs and activities must be established. The respective self-study is carried out over an extended period of time with direct involvement from the entire academic community. To accomplish this, institutions must address internal issues related to structure, process, and governance.

In response to the current self assessment needs [3] the development and implementation of an automated tool that will assist and guide accreditation processed was justified. The system that was developed is called Accreditation Management System (AMS) and aims at addressing both the technology culture and the information culture of an institution. Both cultures are unique to the organizational context of existing universities. Our technology culture is about the use and integration of technology in planning, development, operations, and assessment while the information culture involves information policies and practices for sharing information within and across the organization (like ownership of information).

\section{System Analysis}

The initial phase of the analysis aimed at identifying the requirements that the application should satisfy. A series of interviews with the accreditation steering committee members at the Hellenic American University (HAU) was conducted along with an 
additional set of interviews of outside experts (faculty form other American universities with previous experience in the accreditation process). At the same time a thorough review of the guidelines for accreditation of the six American regional accreditation bodies [4], [5], [6], [7], [8], [9], took place to identify similarities and differences between the various approaches. In addition the newly proposed standards [3] for the EEC countries [10] were taken into consideration.

It was evident from the analysis that a process-based application was required that would promote a collaborative approach to assessment, encouraging broad participation in the assessment process and ensuring reflective analysis of the assessment results. The traditional framework for action in approaching the organization and management of the self study follows certain general principles and procedures. The AMS is organized around a steering committee concept that is composed of a chairperson, chairs responsible for each standard, members, and guests who also play the role of advisors. The chairperson's role is planning and overseeing of the work produced by the rest of the team, suggest approaches and assume the responsibility for having the final report edited. He also reviews the standards for accreditation set by the corresponding quality assurance agency and makes sure that all the ways in which the institution provides its academic program to students are incorporated into the self study.
The chairs of each standard are responsible for organizing the efforts needed to address the requirements of their corresponding standards by assigning duties to members of the team. They report directly to the committee chair. The different roles don't necessary assigned to separate single individuals. For example the committee chair can also be the chair of some standards and a certain standard might have more than one chair. Committee members work to ensure inquisitiveness and reflection and are responsible for gathering information, evidence and data.

The AMS application was broken down into the following subsystems and modules: An authorization subsystem, a progress reporting subsystem, a standard's editing subsystem, a standard's management subsystem, a repository of information (DBMS) module, a server management module and a collaboration/communication module. The difference between the module and subsystem classification is that subsystems can be considered as independent entities that are doing all the intelligence of the application while the modules are functional entities used by many subsystems. As of the time of this writing the repository of information module was Apache-Derby (an open source DBMS) and the communication module was carried out by using regular e-mail messages. The choice of the later was purposefully made because of the wide acceptance and dependency that committee members have of e-mail communication (nowadays

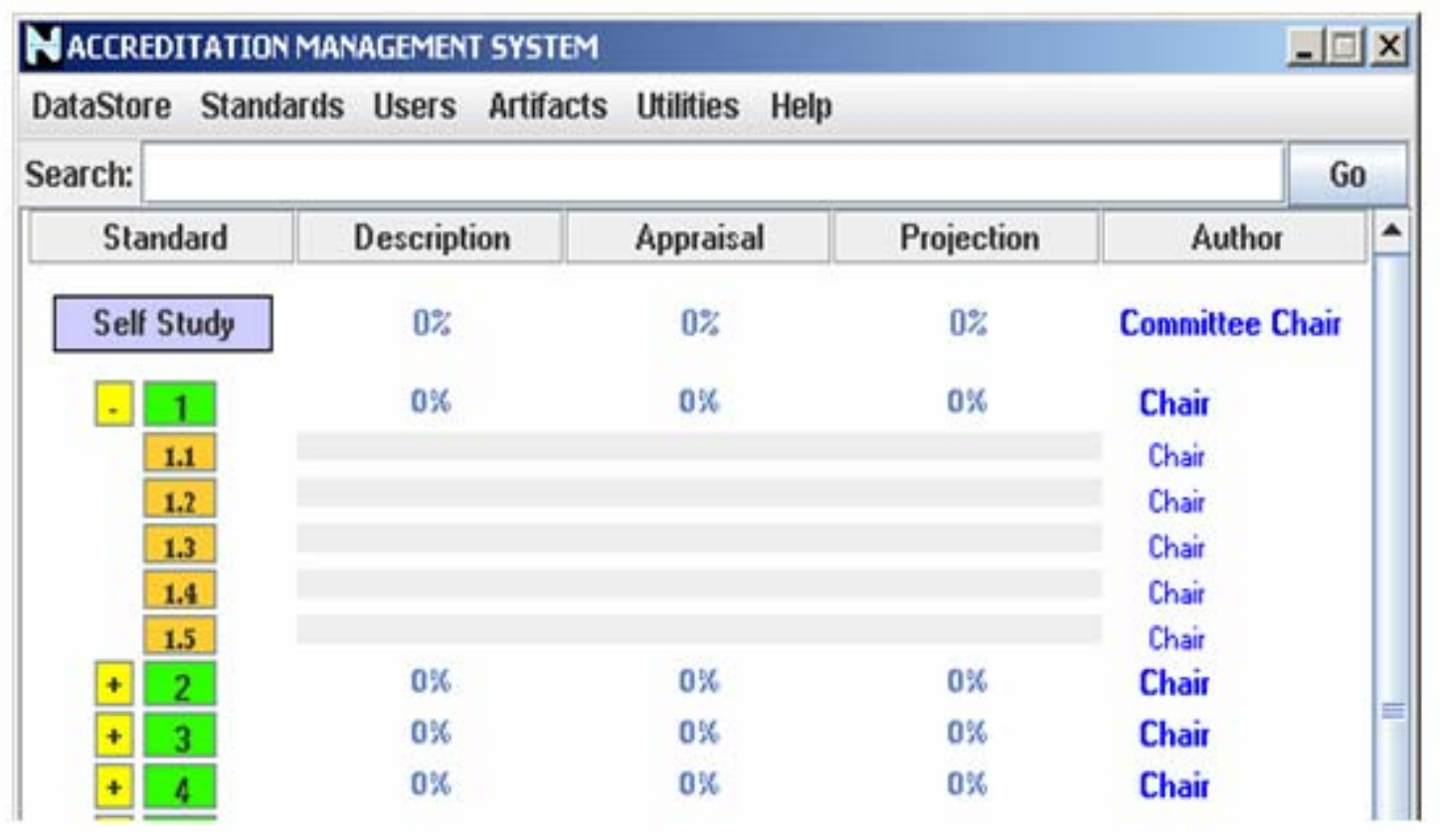

Fig.1 Progress Reporting Window 
everybody checks their e-mail).

\subsection{Repository of Information module}

That is the central repository of any type of information including a variety of document formats, pictures, video and anything that can be launched through a native application. In terms of information content the repository included factual and statistical data. The accreditation process needs to use both existing institutional information and data as well as consider and initiate the gathering of any additional information necessary of useful in the

\subsection{Progress reporting subsystem}

The authorization structure within AMS dictated the progress reporting structure. Member progress is reported to the "standards" chairs that subsequently define the overall progress of the self study (Fig. 1).

\subsection{Standard's management subsystem}

This is the hart of the AMS application. It's the place were standards are broken down in the various sub-standards that can further brake down into individual sentences and phrases and even individual words. The main focus of any

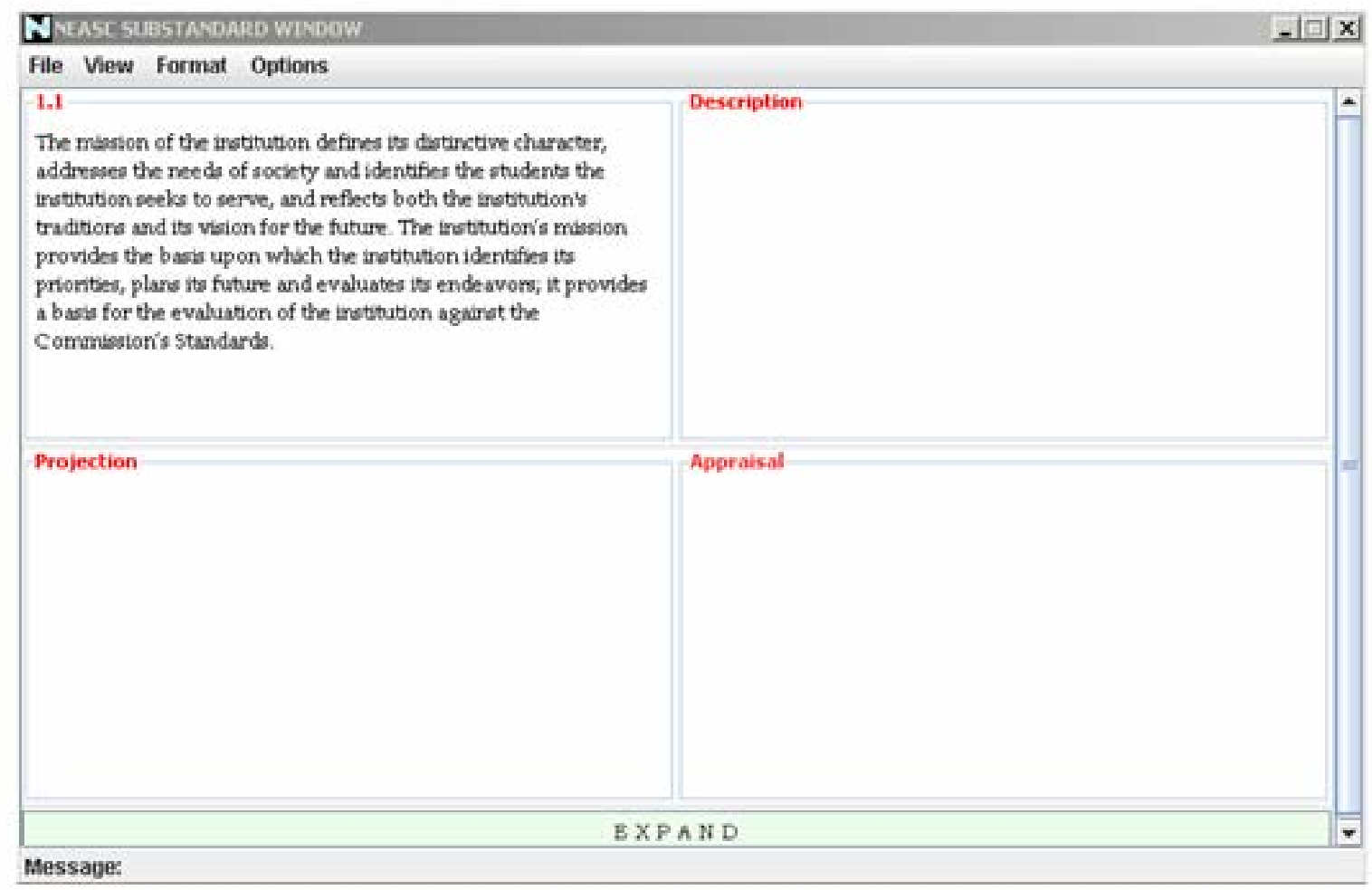

Fig. 2 Standards Management Window

process. The repository is supposed to make the storage and retrieval of information as easy as possible in order to assist team members in grasping meanings and relationships among data.

\subsection{Authorization subsystem}

The distribution of authority within AMS follows the committee structure, with the committee chair authorising "standard" chairs that subsequently authorize members. The committee chair is also responsible for giving "guest" access to anybody he considers could have valuable contribution to the self study. Only "guests" can become committee members by been selected from each "standard's" chair. accreditation process is accountability for the statements and claims made by institutions. Simply stated the institutions need to provide to accrediting agencies their rational for any statements they make concerning the past, present and future of their operation accompanied by corresponding supporting “evidence”.

The subsystem (Fig. 2) is organized around the analytical framework of description, appraisal and projection as suggested by NEASC. This organized triad help group all the possible questions that can be expressed through the six interrogatives of the English language What, When, Who, Where, How and Why. The first five are primarily addressing the statements made by the institution 
and dominate the description and projection sections while the Why is primarily the focus of the appraisal section. The triad is organized in a clock wise cyclical fashion in the application window to represent the flow of time from past, present, and future. More analytically the framework has as
Projection: After the appraisal section the institutions are expected to indicate plans developed to maintain and enhance strengths and address areas of concern. Emphasis on realistic and specific projections should be given that represent a definite commitment to development in the areas addressed

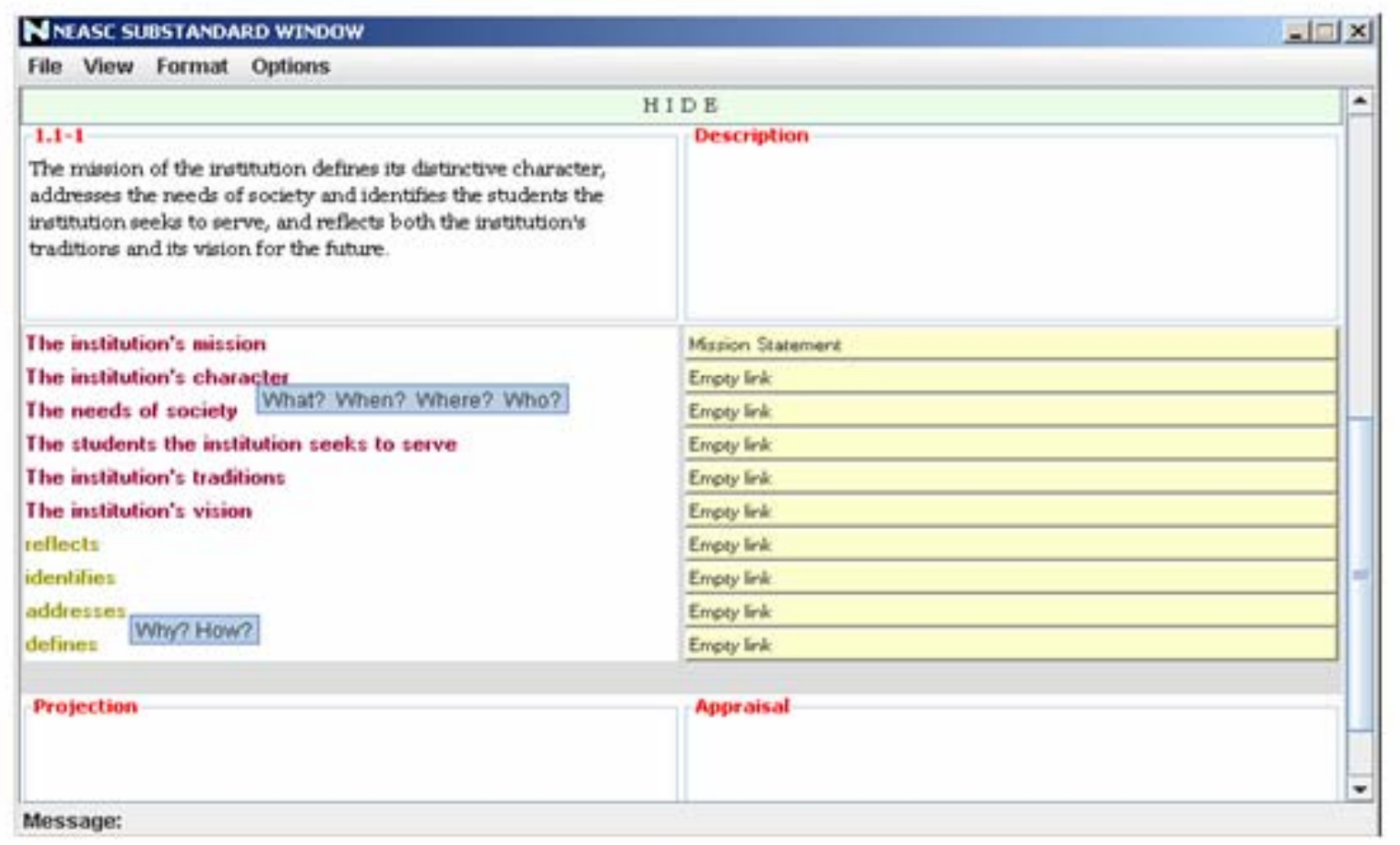

Fig. 3 Deeper level of Sub-Standard Management

follows:

Description: The present status of the university is presented with respect to the adopted standards. A balance is required between providing too much or too little. The various areas of operations need to be presented in a concise way and references or links to existing publications can be included. This section will provide the appropriate groundwork for understanding the concerns raised in the ensuing appraisal and projection sections. In essence, this section sets the stage and provides the context for meaningful analysis of the institution in terms of the various standards.

Appraisal: This is the section were the analysis takes place. The institution is required to make a thorough assessment of the effectiveness of its practices in a given area, recognizing both the institution's achievements and its problems. In this section the institution will summarize its self evaluation as to how well it meets the standard and provide links to sufficient evidence so that the reader of the report understands the basis on which the judgements were made. by the standards in the near future. It is in this section that the results of the self-evaluation are translated into practice.

Given the cyclic nature of the three sections that sort of follow the time continuum of Past, Present and Future the process is expected to offer a complete coverage of the self assessment process. The Description section can be seen as a reference to the Past albeit a very close one, the Present can be expressed through the appraisal section since it represents our current awareness of the situation and the future is represented with the Projection section where future planning and actions are described.

The framework can be repeated at any level in depth until it reaches the literal atomic units that are the words of the text (Fig. 3). In figure 3 we see a division of the substandard of figure 2 into a deeper level that refers to the first sentence of the substandard. Between the framework triad (Description, Appraisal, and Projection) we can see the final breakdown in the literal atomic elements of the text. These are organized in two different categories in order to further assist the user's 
inquisitiveness and offer a complete coverage of every conceivable element of the academic standard.

The first category of literal atomic elements represents the nouns in the text sentence while the second category represents the verbs of the sentence. repository. Being that this subsystem is an editing tool that in many cases will serve as the final editing mechanism of the section of the final report that corresponds to certain standard, emphasis was given to the organization and easy access of any kind of

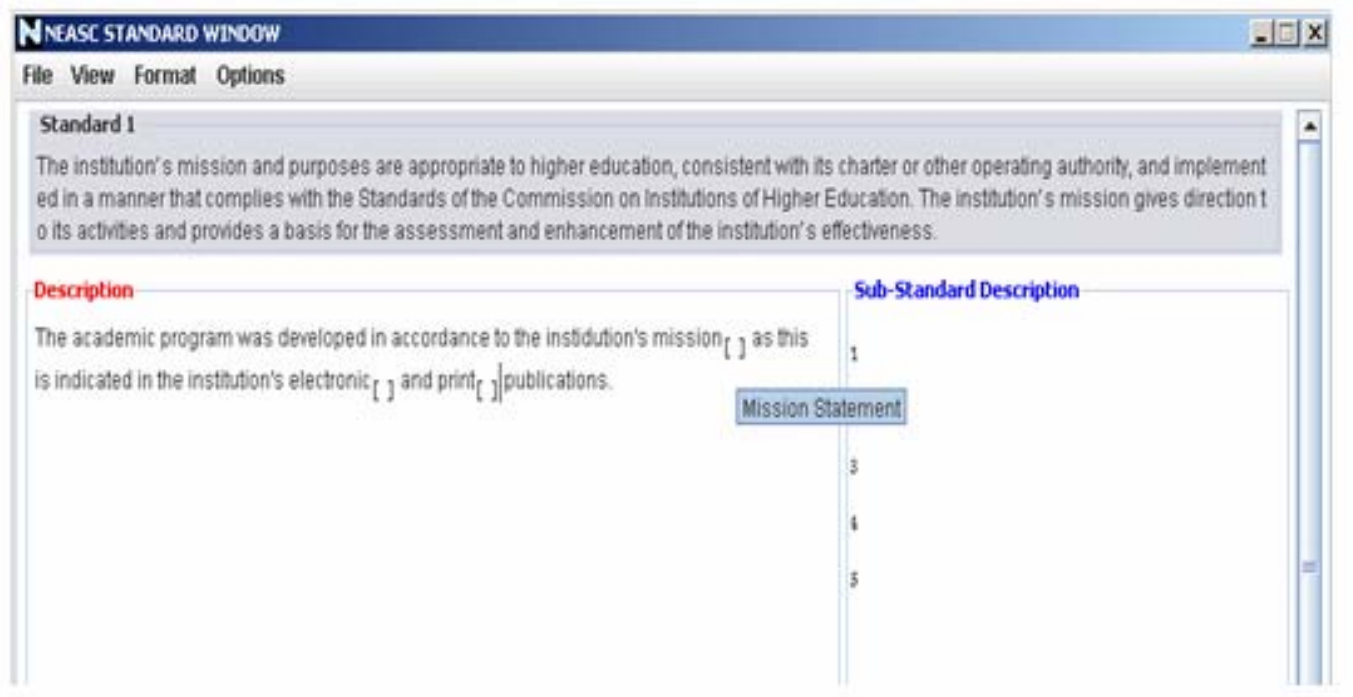

Fig. 4 Standard's Editing Window

Tool tip messages have been implemented to remind the user that nouns help him address questions of What, When, Where, and Who, while verbs (and adverbs) help him provide explanations and rationales through Why and How questions. Since arguments will be supported by evidence users can directly link atomic literals to the information repository. This way at any time they can view the artifact that supports their argument and act accordingly. The environment also offers to the users the ability to link many artifacts (up to 10 at the time of this writing) if they need to do so in support of their arguments.

\subsection{Standard's editing subsystem}

After much deliberation and exchange of ideas, the steering committee members can work in preparing the various sections of what will become the selfstudy report. Ultimately, the work of the committees including their findings will be distilled following the adopted framework and can serve as the basis of the report. The standard's editing subsystem is the obvious place where document editing and manipulation capabilities are included. The most frequently used formatting (font selection, font size, font decoration and color) options have been incorporated.

The users (chairs of standards) also have the ability to insert references of any object in the information that needs to be available. That includes (as it appears on the right side of fig. 4), all the information that is being produced by the members in charge of each substandard.

\section{System Development}

The application was developed using Java 5 for cross platform compatibility reasons and Netbeans 4.1 was the development platform. For database management system I used Apache Derby which is pure Java RDBMS that is part of the Apache foundation as project Derby. Although the tool was modelled along the NEASC specifications it can be easily customized or adapted to any accreditation framework that operates around a "standards" concept. That includes the rest of the US higher education accreditation bodies and the newly proposed European standards.

\section{Conclusions}

Knowledge management in higher education institutions seen as a mix of information practices and learning strategies is gaining wide acceptance. The quality assurance process as expressed through the self evaluation study can be greatly enhanced with a knowledge management tool such as AMS. 
By putting intuition into practice, the AMS builds upon collegial and professional teamwork by actively linking people, processes, and technologies.

AMS eases the rigor of assessment and bolsters institutional improvement efforts by conveying a common understanding of the process, generating uniform report formats for communicating progress, and facilitating a collaborative environment for continuous reflection and improvement. AMS is easy to learn and access offering faculty and staff the ability to document, store, and communicate progress for any standard or substandard they are responsible for.

A major advantage of the AMS is that it will allow the participation of the accrediting body in an institution's accreditation process from the initial phases. This way valuable feedback and guidance can be given to the committee members in advance saving time and energy and producing a more positive outcome.

With the assistance of knowledge management tools such as AMS higher education institutions can focus on promoting policies and practices that help people share and manage knowledge. The final result is a broader knowledge of educational purposes and a more informed understanding of their application to the specific objectives of the institution.

Technology implementation though does not necessarily improve decision-making, nor does it necessarily improve outcomes. Trust in the data produced and commitment in the proper use of systems are necessary ingredients for the success of such systems. To guarantee participation a clear message needs to be conveyed to the users that the primary and only use of the systems is to seek improvements.

\section{Acknowledgments}

Special thanks to my graduate students Basdeli Eleni, Contogiannopoulou Christina, Petrou John, and Sinos Leonidas for their help in the interviewing process.

\section{References:}

[1] Kidwell, J. J., Vander Linde, K. M., Johnson, S. L. "Applying corporate knowledge management practices in higher education,” Educause, 4, 2000, pp. 28-33.

[2] Thorn, C. A., (2001). "Knowledge for educational information systems: What is the state of the field?" Education
Policy Analysis Archives. vol. 9, 47.

[3] Bologna Process (1999), http://www.bologna-bergen2005.no/

[4] Middle States Commission of Higher Education http://www.msche.org/

(2005).

[5] NEASC (New England Association of Schools and Colleges), (2005). http://www.neasc.org/

[6] North Central Association of Colleges and Schools http://www.ncahigherlearningcommission.org/

[7] Northwest Commission on Colleges and Universities (2005), http://www.nwccu.org/

[8] Southern Association of Colleges and Schools (2005), http://www.sacs.org/

[9] WASC (Western Association of Schools and Colleges) (2005), http://www.wascweb.org/

[10] ENQA (European Network for Quality Assurance) (2005), http://www.enqa.net/ 